Journal of Engineering and Applied Sciences 14 (13): 4430-4434, 2019

ISSN: 1816-949X

(C) Medwell Journals, 2019

\title{
Lossy Compression of Hyperspectral Images Using Real-Time Technique
}

\author{
Haitham S. Hasan and Mais A. Alsharqi \\ Department of Business Information Technology, \\ University of Information Technology and Communications, Baghdad, Iraq
}

\begin{abstract}
Several proposed methods related to Hyper-Spectral (HS) image compression have been published in the recent years. These methods have often effective compression accuracy but they are time-consuming. This study introduces the development of a real-time practical scheme for use in lossy HS image compression. This scheme includes two parts; hardware using the Field Programmable Gate Array (FPGA) system and software utilizing the band prediction and fractal encoding techniques. The software technique starts by partitioning the HS image into a number of Groups of Bands ( $\mathrm{GoBs}$ ). Then, the first band in each GoB is utilized by the intra-band prediction to exploit the spatial correlation. And the other bands in each GoB are employed by the inter-band fractal coding technique as well as a limited search algorithm to make a complete benefit from the local matching between any two neighboring bands. This technique shows that the reconstructed image has a better improvement in the classification accuracy than the primary uncompressed image but still time-consuming. So, to overcome this problem, the technique is implemented by using the FPGA. This hardware technique is extremely suitable for real-time purposes.
\end{abstract}

Key words: Fractal encoding, prediction, lossy compression, hyper-spectral image, exploit, technique

\section{INTRODUCTION}

Image spectrometry can obtain spatial and spectrum information of the ground target, concurrently. As a result, a hyper/multi-spectral image is a 3D data cube: 1D in spectrum and 2D in space. Compared to multi-spectral images, HS images have narrower and more contiguous spectral bands of the electromagnetic radiation reflected from the same scene and higher spectral resolution. Generally, HS images contain bands in the hundreds, while multi-spectral images have $<100$ bands. HS images can offer an enhanced diagnostic capability for object identification, classification, detection and tracking than multi-spectral images (Du et al., 2008; Santos et al., 2012). On the other hand with the number of spectral bands growing, HS images result in a very large volume of data. This volume is not suitable for storage, analysis and transmission. Therefore, it's very important to compact HS images proficiently. HS image compression techniques can be categorized into two major categories: lossless and lossy compression approaches. The lossless compression (Mat Noor and Vladimirova, 2013) approach can keep all the original information about the HS image but the compression ratio is minimal which cannot be suitable for many practical purposes. Alternatively, the lossy compression method is utilized to preserve the fundamental spectral information of the HS image.
This attempt is to maintain equilibrium between the information loss and compression efficiency (Zabala and Pons, 2013).

However, there are many methods for lossy HS image compression. JPEG 2000 international standard (Taubman and Marcellin, 2012; Dusselaar and Paul, 2017) and its extension (2004) have been used widely. Karami et al. (2012) proposed an effective approach for HS image compression by the utilization of Discrete Wavelet Transform and Tucker Decomposition (DWT-TD). In addition to transforming-based techniques as mentioned above, predictive coding methods are also used for HS image compression. A lossy compression method with low-complexity is introduced by Abrardo et al. (2011). It is based on rate-distortion optimization and prediction, uniform-threshold quantization. By Miguel et al. (2006), the proposed prediction technique used was linear between the bands where each band, excluding the first band is predicted by the band earlier transmitted. The predictor utilized a bit plane-synchronized closed loop technique. At the encoder, it does not need a complete decompression of the preceding band, so as to decrease the predictive encoder complexity.

Conversely, Zhao et al. (2010) introduced an effective macro-block. It is based different and adaptable prediction modes selection for hyper-spectral images encoding. It is motivated by the prediction system of H.264/AVC video

Corresponding Author: Haitham S. Hasan, Department of Business Information Technology, University of Information Technology and Communications, Baghdad, Iraq 
coding standard (Wiegand et al., 2003). The best possible reference band is decided through a band selection algorithm. Subsequently, the most excellent partition of the candidate macro-block in the best possible reference band is extra chosen for inter-band prediction of the present macro-block. This technique does better than the up to date 3D-based compression algorithms at worse percentages and about $80 \%$ of the encoding time can be saved compared with the technique by employing the whole prediction modes of H.264/AVC. A performance assessment of the up to date H.264/AVC video coding standard is accomplished by Marcellin (2004) and Miguel et al. (2006) and the objective of verifying its viability when employed to HS image compression. Simulations achieve compression ratios up to 0.13 bits per pixel per band (bpppb) for real HS images.

Compared to the algorithms above, fractal coding is a comparatively different idea of compressing images by using the local self-similarity in an image. It has the advantage of outcome self-determination, high compression ratio and fast decoding. Consequently, it is regarded as a capable compression technique. The original fractal image coding scheme is produced by Barnsley in which the transform consists of the affine mappings number union of the entire image. Actually, it is an Iterated Function System (IFS) and the encoding process can't be done automatically (Wohlberg and de Jager, 1999). For example, the Partitioned IFS (PIFS) by Jacquin (1992, 1993), each of the individual mappings motivates on the image subset instead of the whole image. The fractal compression was converted into a practicable truth and the standard algorithm is formed to code fractal block. A bit rate $<1$ bit/pixel can be achieved by such a system aimed at the coding of black and white digital images exclusive of any entropy coding of the fractal code parameters. This system demonstrates the fractal coding which has an extremely high compression-ratio. Unfortunately, the main disadvantage is that the process of the block similarity is extremely time-long. To reduce the matching complexity, many efforts have been made to get it faster. Among these efforts, a valuable technique is to reduce the searching process by eliminating several of the domain blocks, since, most of them are not used in the normal fractal coding (Jacobs et al., 1992; Tong and Pi, 2001; Wan and Hsieh, 2001; Tong and Wong, 2002).

Finally, Zhao et al. (2016) developed an appropriate new method by utilizing both the inter-band fractal and the intra-band prediction coding techniques. Initially, the methodology starts by partitioning the HS image into a number of Groups of Bands (GoBs). The fractal encoding or prediction method is selected for the various bands as well. Then, a sign Exp-Golomb entropy encoded is given to the whole fractal parameters. Lastly, the compensation mechanism is used. This technique achieves the highest compression efficiency among the other available techniques. Also, it has better improved classification accuracy related to the reconstructed image than the original one. The main drawback of this technique is still time-consuming. So, to overcome this problem, the technique is implemented by using a hardware system called the Field Programmable Gate Array (FPGA). The type of the used FPGA system is Xilinx Spartan 3A. This hardware technique is extremely suitable for real-time purposes.

\section{MATERIALS AND METHODS}

As mentioned above, the HS image structure is a 3D data, encapsulating spectral and spatial information. The process of HS image compression includes two key steps. The first step is an extremely significant step to remove spectral redundancy. Generally, it is spectral decorrelation (or so called "inter-band compression") and it is working on 1D reduction. The second step concentrates on several types of compression encoders. It is identified as intra-band compression technique. It is a 2 or $3 \mathrm{D}$ encoder (Dusselaar and Paul, 2017). In this study, the implemented approach is suggested by Zhao et al. (2016) as illustrated in Fig. 1.

Initially, HS bands are divided into many GoBs. Next, all bands are partitioned into $\mathrm{R}$ blocks each. The size of each block is $16 \times 16$. The first band in a GoB is identified as "I-band" while the others are identified as "P-band". Intra-band prediction is used for I-band only as each I-band pixel is predicted by the adjacent decoded pixels. Inter-band fractal encoding is employed for P-band as each block (in the range) will achieve its greatest similar block (in the domain) from the related reference band in the previous P-band or I-band. It should be noted to use only the previous adjacent band for encoding the current P-band, (Fig. 2) (Zhao et al., 2016). Also, the GoB size is verified at the encoding procedure end due to the ability of utilizing spatial correlation through intra-band reduction and the local match between the neighboring bands through the inter-band fractal encoding.

The second step is to select the technique used for the current range block based on its location into I-band or P-band. Intra-band prediction is used only for I-band. The predictive error will be larger if $1 \mathrm{D}$ intra-band prediction is used, due to the fact that the HS image is usually texture abundant. Applying multi-direction prediction is a better solution to enhance the compression efficiency. Therefore, H.264/AVC is used as a reference. A 


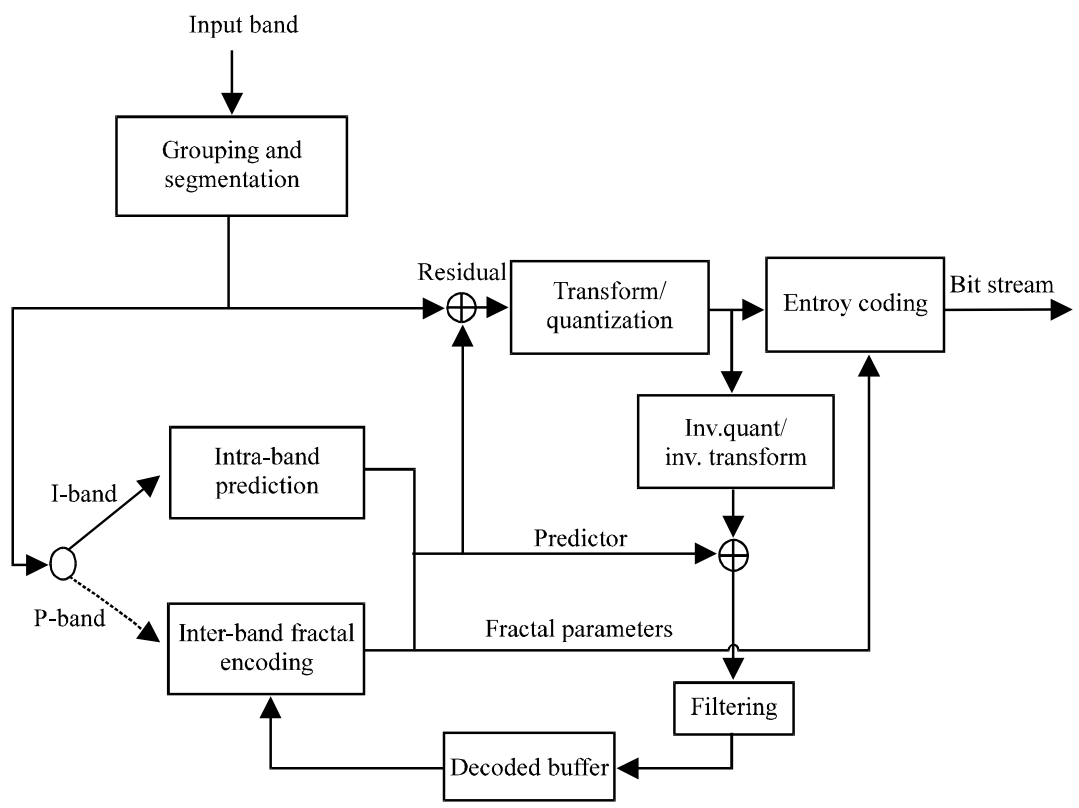

Fig. 1: The compression approach block diagram (Zhao et al., 2016)

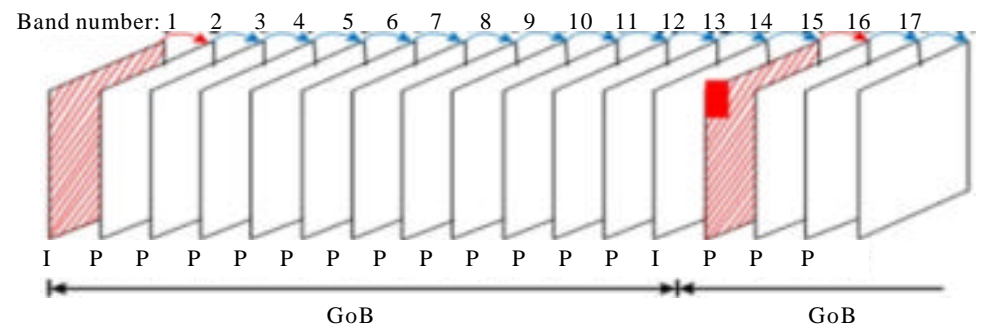

Fig. 2. Band grouping and frame classification (Zhao et al., 2016)

block of size $16 \times 16$ is the basic unit of H.264/AVC. Also, it can be divided into sixteen sub-blocks of size $4 \times 4$. Four types of prediction modes are there for the $16 \times 16$ block, while for the $4 \times 4$ sub-block, there are nine types of prediction modes. Each block is checked for all feasible prediction modes and the rate distortion optimization is utilized to choose the best one so as to get the optimal coding efficiency. In this study, only $4 \times 4$ prediction modes are used, so as to prevent the utilization of the low correlated pixels in prediction of the current block.

In contrast, fractal compression is working on the basis of the local matching exist in the image. For instance, the divided range block has to look for the similar domain block placed in the domain blocks of the image. It is extremely time consuming. A complete utilization of the local matching is made between two adjacent bands and by using the fact that "the two blocks located in the same position of two adjacent bands are highly similar" (Zhao et al., 2016). However, the most popular partition mechanism uses a quad-tree scheme (Aswani and Kamble, 2014): a $\mathrm{R}^{2}$ block is recursively broken up into four quadrants until the resulting blocks are considered covered well enough by a domain block. In this study, tree structure partition is used instead. It can reduce the number of blocks compared with the quad-tree partition. Zhao et al. (2016). The next step is to sign the fractal parameters with Exp-Golomb entropy coding, according to the conditions stated by Zhao et al. (2016). This step enables the fractal parameters to encode by a smaller number of bits compared with the traditional entropy coding method.

The final step is the error compensation process. It is added to enhance the HS image decoded quality. Both the fractal-residual and the prediction error are extra quantized, transformed and entropy encoded (Zhao et al., 2016). Integral DCT and a combination of the DCT with quantization are used for simplicity and efficiency. 


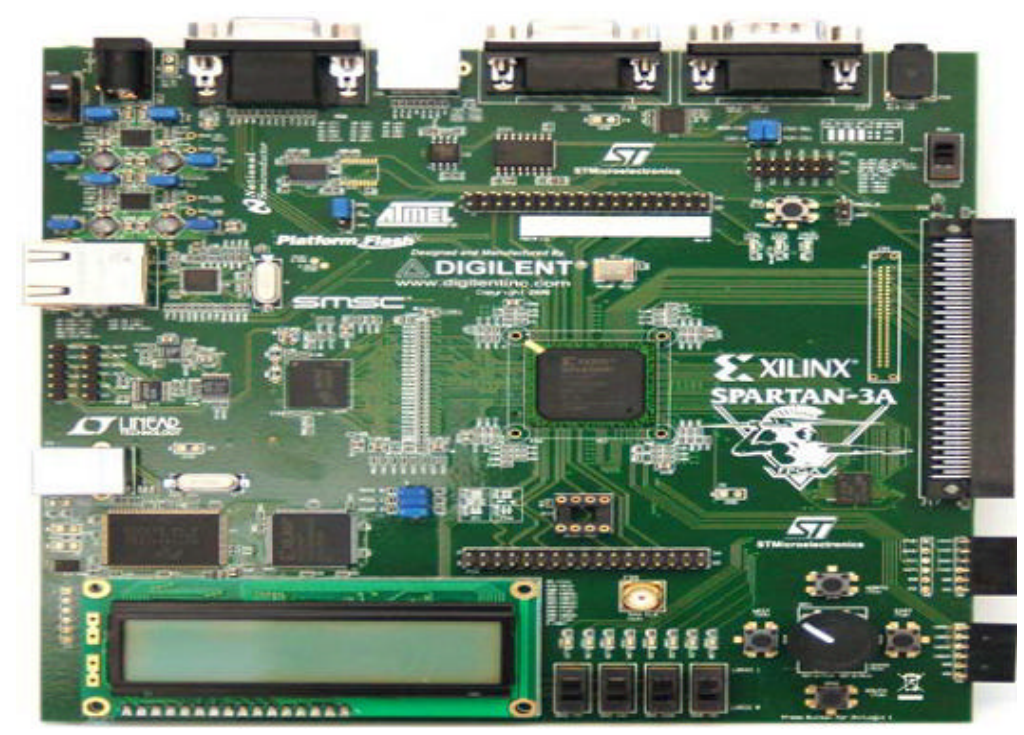

Fig. 3: Hardware side view

Therefore, there is a need only for executing bit-shifts, multiplications as well as additions in the whole transformation and quantization process. Consequently, the coefficients of the quantization process are rearranged by zig-zag scan. Next, these coefficients are entropy encoded by Context Adaptive Binary Arithmetic Coding (CABAC). The final decoded block is achieved by inverse transformation. This transformation is performed by "inverse quantization of the residual $\mathrm{R}$ block and adding to the intra-band predicted $\mathrm{R}$ block or the inter-band fractal iterated decoded R block" (Zhao et al., 2016) (Fig. 3).

\section{RESULTS AND DISCUSSION}

The evaluation of the system performance is based on the compression efficiency, classification accuracy and the processing time. Lunar Lake, Jasper Ridge and Cuprite are three AVIRIS images (Anonymous, 2018) used in the encoding experiments. These images consist of 224 bands and the size of $512 \times 512$ in space. The results show that the software scheme has a tendency to do better as the GoB size Grows to be Bigger. Also, the gained PSNR is bigger compared to other methods with a bit rate $>0.4$ bpppb. And the classification accuracy is better than the available innovation compression methods (Zhao et al., 2016).

From the processing side view, the software was processed on a computer with Intel Core i7, $2130 \mathrm{MHz}$ CPU, $6 \mathrm{MB} \mathrm{L} 3$ cache and $8000 \mathrm{MB}$ of RAM. To overcome the time-consuming problem, the software is implemented on FPGA Xilinx-ISE 14.7 with Spartan-3A XC3S700A board which offers an exceptional tool for mixing between the MATLAB R2013a and ISE 14.6 (2013) platforms. MATLAB offers components that use Verilog code of Xilinx platform for avoiding the weakness of XC3S1400A-FPGA board size.

\section{CONCLUSION}

This study is a real-time technique for use in lossy compression of HS images. The implementation of the technique consists of two parts; hardware using FPGA system and software sing lossy compression method. The type of the FPGA system is Xilinx Spartan 3A. The software is based on lossy compression method through the utilization of the intra-band prediction and inter-band fractal encoding techniques. To decrease complexity, an algorithm of local search is developed to get free of the comprehensive search and the isometric transformation operations. The results show that the reconstructed image has improved classification accuracy with an unbelievable reduction in the HS image compression time. The paper has a significant contribution in the real-time HS compression. It provides a new view for HS image compression on the basis of the processing time as well as the compression efficiency.

\section{REFERENCES}

Abrardo, A., M. Barni and E. Magli, 2011. Low-complexity predictive lossy compression of hyperspectral and ultraspectral images. Proceedings of the 2011 IEEE International Conference on Acoustics, Speech and Signal Processing (ICASSP), May 22-27, 2011, IEEE, Prague, CzechRepublic, ISBN:978-1-4577-0538-0, pp: 797-800. 
Anonymous, 2018. Aviris; Airborne visible/Infared imaging spectrometer. Aviris, Europe. https://aviris.jpl.nasa.gov/

Aswani, R.A. and S.D. Kamble, 2014. Fractal video compression using block matching motion estimation-a study. J. VLSI. Signal Process., 4: 82-90.

Du, Q., N. Raksuntorn, S. Cai and R.J. Moorhead, 2008. Color display for hyperspectral imagery. IEEE. Trans. Geosci. Remote Sens., 46: 1858-1866.

Dusselaar, R. and M. Paul, 2017. Hyperspectral image compression approaches: Opportunities, challenges and future directions: Discussion. J. Opt. Soc. Am. A., 34: $2170-2180$.

Jacobs, E.W., Y. Fisher and R.D. Boss, 1992. Image compression: A study of the iterated transform method. Signal Process., 29: 251-263.

Jacquin, A.E., 1992. Image coding based on a fractal theory of iterated contractive image transformations. IEEE Trans. Image Process., 1: 18-30.

Jacquin, A.E., 1993. Fractal image coding: A review. Proc. IEEE., 81: 1451-1465.

Karami, A., M. Yazdi and G. Mercier, 2012. Compression of hyperspectral images using discerete wavelet transform and tucker decomposition. IEEE. J. Sel. Top. Appl. Earth Obs. Remote Sens., 5: 444-450.

Marcellin, M.W., 2004. ISO/IEC 15444-2:2004, Information technology-JPEG 2000 image coding system: Extensions. 1st Edn., ISO/IEC, New York, USA., Pages: 321 .

Mat Noor, N.R. and T. Vladimirova, 2013. Investigation into lossless hyperspectral image compression for satellite remote sensing. Intl. J. Remote Sens., 34: 5072-5104.

Miguel, A.C., R.E. Ladner, E.A. Riskin, S. Hauck and D.K. Barney et al., 2006. Predictive Coding of Hyperspectral Images. In: Hyperspectral Data Compression, Motta, G., Rizzo, F. and Storer, J.A. (Eds.). Springer, Boston, Massachusetts, USA., ISBN:978-0-387-28579-5, pp: 197-231.
Santos, L., S. Lopez, G.M. Callico, J.F. Lopez and R. Sarmiento, 2012. Performance evaluation of the H.264/AVC video coding standard for lossy hyperspectral image compression. IEEE. J. Sel. Top. Appl. Obs. Remote Sens., 5: 451-461.

Taubman, D.S. and W.M. Marcellin, 2012. JPEG2000 Image Compression Fundamentals, Standards and Practice. Springer, Berlin, Germany, ISBN:978-1-46135245-7, Pages: 767.

Tong, C.S. and M. Pi, 2001. Fast fractal image encoding based on adaptive search. IEEE. Trans. Image Process., 10: 1269-1277.

Tong, C.S. and M. Wong, 2002. Adaptive approximate nearest neighbor search for fractal image compression. IEEE Trans. Image Process., 11: 605615.

Wan, C.C. and C.H. Hsieh, 2001. An efficient fractal image-coding method using interblock correlation search. IEEE. Trans. Circuits Syst. Video Technol., 11: 257-261.

Wiegand, T., G.J. Sullivan, G. Bjontegaard and A. Luthra, 2003. of the H.264/AVC video coding standard.IEEE Trans. Circuits Syst. Video Technol., 13: $560-576$.

Wohlberg, B. and G. de Jager, 1999. A review of the fractal image coding literature. IEEE Trans. Image Process., 8: 1716-1729.

Zabala, A. and X. Pons, 2013. Impact of lossy compression on mapping crop areas from remote sensing. Intl. J. Remote Sens., 34: 2796-2813.

Zhao, D., S. Zhu and F. Wang, 2016. Lossy hyperspectral image compression based on intra-band prediction and inter-band fractal encoding. Comput. Electr. Eng., 54: 494-505.

Zhao, F., G. Liu and X. Wang, 2010. An efficient macroblock-based diverse and flexible prediction modes selection for hyperspectral images coding. Signal Proc. Image Commun., 25: 697-708. 